\title{
Quantitative Analysis of Localized Changes in Breast Shape
}

\author{
Audrey L. CHEONG ${ }^{1}$, Gregory P. REECE ${ }^{2}$, Mia K. MARKEY ${ }^{3,4}$, \\ Michelle C. FINGERET ${ }^{2,5}$, Fatima A. MERCHANT'1,6 \\ ${ }^{1}$ Department of Electrical and Computer Engineering, University of Houston, Houston TX, USA; \\ ${ }^{2}$ Department of Plastic Surgery, The University of Texas MD Anderson Cancer Center, Houston TX, USA; \\ ${ }^{3}$ Department of Biomedical Engineering, The University of Texas at Austin, Austin TX, USA; \\ ${ }^{4}$ Department of Imaging Physics, The University of Texas MD Anderson Cancer Center, Houston TX, USA; \\ ${ }^{5}$ Department of Behavioral Science, The University of Texas MD Anderson Cancer Center, Houston TX, USA; \\ ${ }^{6}$ Department of Engineering Technology, University of Houston, Houston TX, USA
}

DOI: $10.15221 / 17.130 \quad$ http://dx.doi.org/10.15221/17.130

\begin{abstract}
Breast reconstruction surgery is an integral part of breast cancer treatment for many patients and has been shown to positively influence patients' psychosocial adjustment and quality of life. Threedimensional (3D) visualization and quantification of the breast for different types of reconstruction procedures during the reconstruction process can allow for a better understanding of the changes in breast shape. The reconstruction process, which involves multiple procedures, can take as long as 18 - 24 months, and the breasts can change shape throughout this period. In this study, we present a novel approach for monitoring and quantifying changes in breast shape using 3D surface images of the torso in conjunction with surface normal measurements. The results of this study can help provide information to physicians and patients about the dynamic changes in breast morphology occurring at different stages of the reconstruction process. Additionally, this information may assist in pre-treatment surgical planning and training. In our approach, we compare the surface normal values of different regions of the breasts at different time-points using the Bhattacharyya distance, which measures histogram similarity. Results are shown for 17 patients who underwent autologous reconstruction, 14 patients who underwent implant-based reconstruction, and one patient who underwent a mixed reconstruction. Using the proposed method, we were able to evaluate changes in the 3D surface images of reconstructed breasts relative to the $3 \mathrm{D}$ images of preoperative breasts.
\end{abstract}

Keywords: 3D image, breast reconstruction, surface normal, shape analysis, quantitative analysis, longitudinal

\section{Introduction}

Breast cancer is one of the most commonly diagnosed cancers among American women. Breast cancer treatment typically involves complete or partial removal of the breast(s) resulting in permanent deformation of the breast. These bodily changes can affect both self-esteem and body image of breast cancer survivors. For many women, breast reconstruction is an important part of the treatment process to improve quality of life.

Breast reconstruction surgery can be broadly categorized into autologous and implant-based reconstructions. The transverse rectus abdominis myocutaneous (TRAM) flap procedure is the most commonly performed autologous procedure. Relative to implant-based reconstructions, TRAM procedures are more likely to result in reconstructed breasts that look and feel more natural, although there is generally more scarring than with implant-based reconstruction. Implant-based reconstruction leads to a more rounded breast shape.

The reconstruction process typically involves multiple procedures or surgeries. The total length of this process may occur over a year or more, since it generally takes about a month to recover after any given surgical procedure, with additional time later as the breast tissue settles to a stable form. Sometimes, the contralateral natural breast undergoes a surgical procedure in order to achieve breast symmetry. It is important to recognize that there is not a breast reconstruction type that suits every patient and the breast reconstruction type that will give the best results varies from person to person. There are many factors that play a role in the overall aesthetic outcome including medical history, anatomy, body shape, smoking history, and body weight. The breast shape may also vary naturally over time due to the menstrual cycle [1].

Our goal in this study is to develop objective measurements that can evaluate the shape characteristics of breasts undergoing reconstruction as well as differences between the two main breast reconstruction types: autologous and implant-based. In this study, we present an approach for visualizing and 
quantifying 3D changes in breast morphology across time for women undergoing breast reconstruction. This information will allow a better understanding of the changes that occur in breast shape throughout the reconstruction process and the resulting outcome.

\section{Related Work}

There is a rich body of prior work on subjective and objective evaluation of breast shape, size, symmetry, and ptosis [2] for surgical planning and outcome assessment. Many studies have relied on in-person measurements and 2D photographs [3, 4] while recent studies have been able to use three-dimensional imaging technologies to automatically extract objective 3D measurements through algorithm development. Earlier studies recorded breast anthropometric measurements for different population groups [5-8] using tape measures, but these can now be more efficiently measured from 3D photos [9], [10]. Other studies with 3D imaging technologies have also assessed breast shape and size. These include studies that use the root mean square (RMS) difference between the breast surfaces to compare shapes [11-13]. Moyer et al. evaluated breast symmetry on breast conservation therapy patients based on breast volume and the RMS value [11]. Losken et al. used the sternal notch to nipple distance and the RMS value to evaluate natural breast asymmetry [12]. While some studies have measured the volume and surface area in different regions of the breast [13, 14], others have compared the upper pole and lower pole (breast area above and below the nipple, respectively) breast volume $[14,15]$. Another study suggested dividing the breast into four quadrants and measuring the surface area of each quadrant [16]. The 2D Percentage Breast Retraction Assessment (pBRA) index [17] is a measure for evaluating breast symmetry, which we extended to a 3D pBRA [18] index.

Previous studies have further used the breast measurements to analyze breast shape changes before and after breast surgery. Eder et al. [14] analyzed breast morphological changes after inverted T-scar and vertical-scar reduction mammoplasty. They observed that the breast shape stabilizes after 3-6 months in the T-scar group and after 6-9 months in the vertical-scar group based on linear measurements (i.e., sternal notch to nipple distance) and the breast contour deviation (breast surface difference between a reference scan and a post-op scan). They also noticed a reduction in breast volume in the first 3 months following surgery, with the breast volume stabilizing afterwards. The breast volume distribution, over time, shifted from the upper pole to the lower pole for both T-scar and verticalscar and stabilized after 6 months and 9 months, respectively. In another study, Eder et al. [19] concluded that after subpectoral breast augmentation, the breast shape stabilizes after 3 months.

This is the first study that allows a quantitative analysis of local breast morphological changes using measures of orientation. We extracted orientation changes in different sections of the breast in order to evaluate changes in breast shape over time during the reconstruction process.

\section{Study Sample}

Three-dimensional surface images of female breast cancer patients (IRB approved) undergoing mastectomy and reconstruction procedures from 2008 to 2016 at The University of Texas MD Anderson Cancer Center were obtained using the 3dMDTorso System (3dMD LLC, Atlanta, GA). All patients were standing in an upright position when imaged. The cameras captured the frontal portion of the torso from the sternal notch to the umbilicus. The patients were generally but not consistently imaged pre- and post-operatively at three month intervals, for up to two years. These images are in a triangular mesh format consisting of 3D points, faces, and texture (color).

Patients who had at least three images from different visits and met the following criteria were selected for this study. The first image (baseline) had to be a preoperative image (before mastectomy). The patients may have received biopsies before the preoperative image was taken. The second image could be a tissue expander or the reconstructed breast (either autologous or implant-based). The third and fourth visit images include reconstructed breast(s), which may have had breast revision surgery. Patients with unilateral and/or bilateral reconstructions were included. This yielded 17 patients who underwent autologous reconstruction, 14 patients who underwent implant-based reconstruction, and 1 patient who underwent both types of reconstruction (an autologous procedure on one breast and an implant-based procedure on the other breast). In total, there were 28 autologous reconstructed breasts and 22 implant-based reconstructed breasts. Since radiation can affect breast shape, we report that four patients (six breasts) undergoing autologous reconstruction had prior radiation therapy, 26 patients did not have prior radiation therapy, and the remaining two patients did not report whether they had radiation therapy. 
The breasts were separated into five different groups as shown in Table 1. The first group contained the preoperative breasts that did not receive any procedures that would modify their shape. Once the preoperative breasts underwent a procedure (e.g., mastopexy), the images taken after the procedure were removed. The second group of breasts underwent a tissue expander placement and then a TRAM flap reconstruction, while the third group of breasts underwent a tissue expander placement and then changed to a permanent implant. These two groups were created to see how the tissue expander changes the shape of the breast. The fourth and fifth groups contained all the TRAM flap reconstruction images and the implant-based reconstruction images, respectively, without regard as to whether a tissue expander was inserted or not. In this way, we can include all the patients with TRAM flap reconstructions that did not use a tissue expander.

Since the patients were not consistently imaged every three months, the sample size for each visit varies (see Table 1). Additionally, implant-based reconstruction usually involves a tissue expander (TE) placement, while most TRAM flap reconstructions do not use a tissue expander. Therefore, the TE to TRAM group has a much smaller sample size than the other groups. All implant-based reconstructions, in this case, included a tissue expander. Each visit consists of images that are matched by the average number of days ( \pm 3 months) after a particular procedure (tissue expander placement, TRAM procedure, or implant-based procedure).

Table 1. Sample size of each group for a particular visit. The first visit are all preoperative images.

\begin{tabular}{|l|c|c|c|c|}
\hline Groups & Visit 1 & Visit 2 & Visit 3 & Visit 4 \\
\hline 1) Preoperative (Pre-Op) breasts & 64 & 14 & 8 & 3 \\
\hline 2) Pre-Op to TE to TRAM & 5 & 5 (TE) & 3 (TRAM) & 4 (TRAM) \\
\hline 3) Pre-Op to TE to implant & 23 & 20 (TE) & 16 (implant) & 12 (implant) \\
\hline 4) Pre-Op to TRAM (w/o TE) & 26 & 23 (TRAM) & 19 (TRAM) & 7 (TRAM) \\
\hline 5) Pre-Op to implant (w/o TE) & 23 & 13 (implant) & 13 (implant) & 9 (implant) \\
\hline
\end{tabular}

At the start of the study, the median age of the selected study sample was 49 years (range: 24 to 66 years) and the median BMI was 27.6 (range: 18.5 to 40.9). There were 26 Caucasians, 3 African Americans, 1 Asian, and 2 classified as Other Race. In terms of ethnicity, 9 patients were Hispanic, 22 patients were not Hispanic, and 1 patient did not specify her ethnicity.

\section{Methodology}

\subsection{Image Cropping}

To extract the left and right breast surfaces from the torso image, we first identified the following fiducial points: the sternal notch, the anterior axillary points, the lateral points, the midline, and the lowest visible point of each breast. Next, we manually selected four points for each breast: the sternal notch, the anterior axillary point, a point behind the lateral point and below the lowest visible point of the breast, and a point along the midline and just below the lowest visible point of the breast as shown in Figure 1.

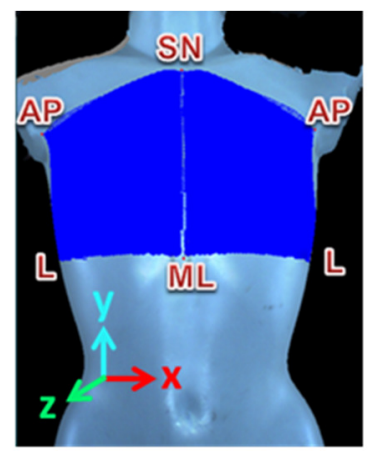

Fig. 1. Image of a torso with the selected left and right breast region highlighted in solid blue, which are cropped.

The Dijkstra's algorithm is used to connect these four points forming the border of the breast extraction. The left and right breasts are analyzed individually, and the left breasts were mirrored to match the right breasts for group analysis. 


\subsection{Surface Normal Analysis}

Our approach was to evaluate the surface normal of each 3D point on the surface mesh with respect to a forward-pointing vector (positive $z$ direction), which is referred to as surface normal orientation angles. These angles ranged from 0 to 180 degrees. As demonstrated for a 3D image of a Styrofoam half sphere (see Figure 2), the surface normal orientation angles calculated at the center of the half sphere were close to zero, and the angles increased towards 90 degrees when moving away from the center towards the peripheral borders. Outside of the half sphere was a flat plane where the angles were zero. In order to determine the surface orientations along the breast mound, we divided a breast into regions as follows. Four concentric rings of increasing radii were created with the diameter of the outermost ring equal to the breast width increased by $12.5 \%$ to ensure that the entire breast mound was included in the analysis. We also make sectional divisions at intervals of 45 degrees, for a total of eight angular divisions. This resulted in segmenting the cropped area enclosing the breast into 32 regional sections. We can describe these divisions in terms of four clinical breast quadrants (upperouter quadrant, upper-inner quadrant, lower-inner quadrant, and lower-outer quadrant) where each quadrant is subdivided into eight regions as shown in Figure 3 . The nipple usually served as a reference point when dividing the breast mound into quadrants. However, since the nipple may be absent in reconstructed breasts, the bottom of the outermost ring was aligned with the lower breast border, and the center of the annular rings was used as the reference point. We then computed two parameters for each region to assess changes in the overall and the local breast shape. For estimating changes in the overall shape of the breast mound, we generated a histogram of the surface normal orientation angles (40 bins equally divided from 0 to 180 degrees) for each region and normalized each histogram to a sum of one. These regional histograms were concatenated providing an estimate of the overall breast shape. Histogram matching with the Bhattacharyya distance [20] as the similarity metric (0 is an exact match and 1 is mismatch) was then used to compare the overall shape of any two breasts. Local breast shape was estimated by computing the average value of the surface normal orientation angles for each region. The Student's t-test with a 95\% confidence interval and the null hypothesis for equality was used to assess significance when comparing the overall or local shape across different reconstructive types (i.e., TRAM flap and implant).

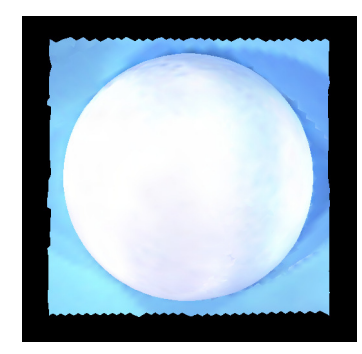

(A)

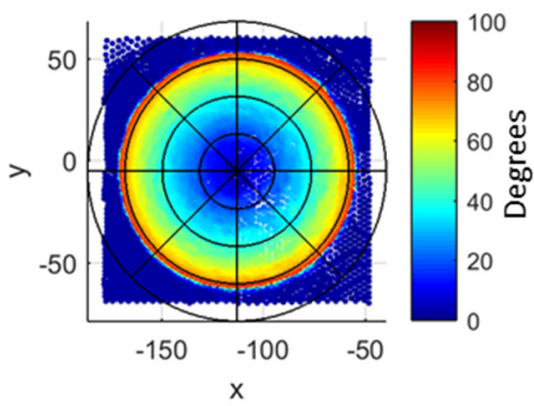

(B)

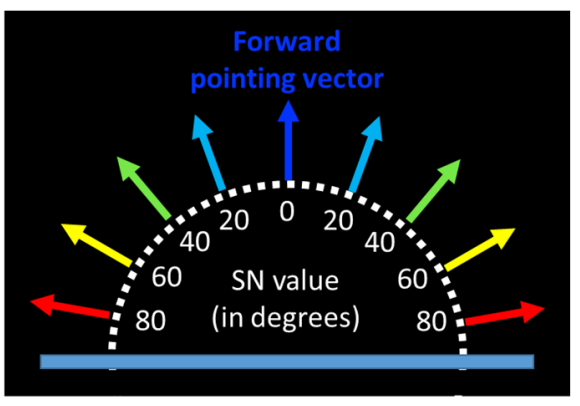

(C)

Fig. 2. A representation of our method. A) Here we have a 3D surface image of a half sphere lying on flat plane (top view) and b) the resulting values using our method on the half sphere. The concentric rings are centered on the most projecting point of the sphere (the center of the image). There are 32 regions created by four concentric rings and the angular divisions at intervals of 45 degrees. C) An example of how the values are calculated for a single slice of the half sphere is shown (side view).

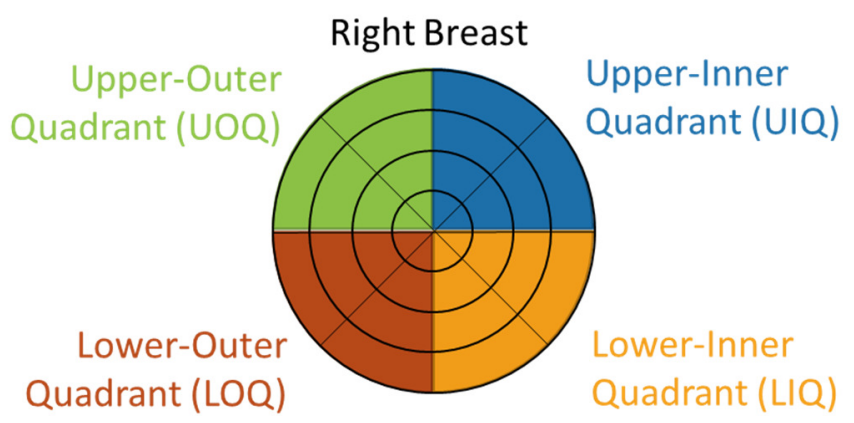

Fig. 3. Four breast quadrants and the regional divisions.

The left breasts were mirrored to match the right breast quadrants. 


\section{Results}

The computed surface normal orientation angles were color mapped onto the 3D surface images to enable a qualitative visual analysis of changes in breast shape. Figure 4 presents representative images from a patient who underwent unilateral TRAM flap surgery on the right breast. As seen, for the preoperative left breast, the distribution of the orientation angles is similar across the different visits (24). In contrast, the reconstructed right breast (visit 2) has distinctly higher values along the boundary of the breast mound, presenting a clear delineation of the rounded shape of the inserted tissue expander (TE). Moreover, subsequent to TE removal, a gradual decrease in surface orientation is observed along the upper pole of the reconstructed right breast in visits 3 and 4 as the reconstructed breast stabilizes its shape following TRAM flap reconstruction.

Figure 5 presents representative images from a patient who underwent a bilateral implant-based reconstruction. For visit 2, the rounded shape of the tissue expander insert is clearly delineated in both breasts with high surface orientation angles, as seen previously in Figure 4. However, in contrast to the TRAM flap reconstruction in Figure 4, we see that the rounded shape of the breast is retained in visits 3 and 4 following exchange of the TE insert by the implant.

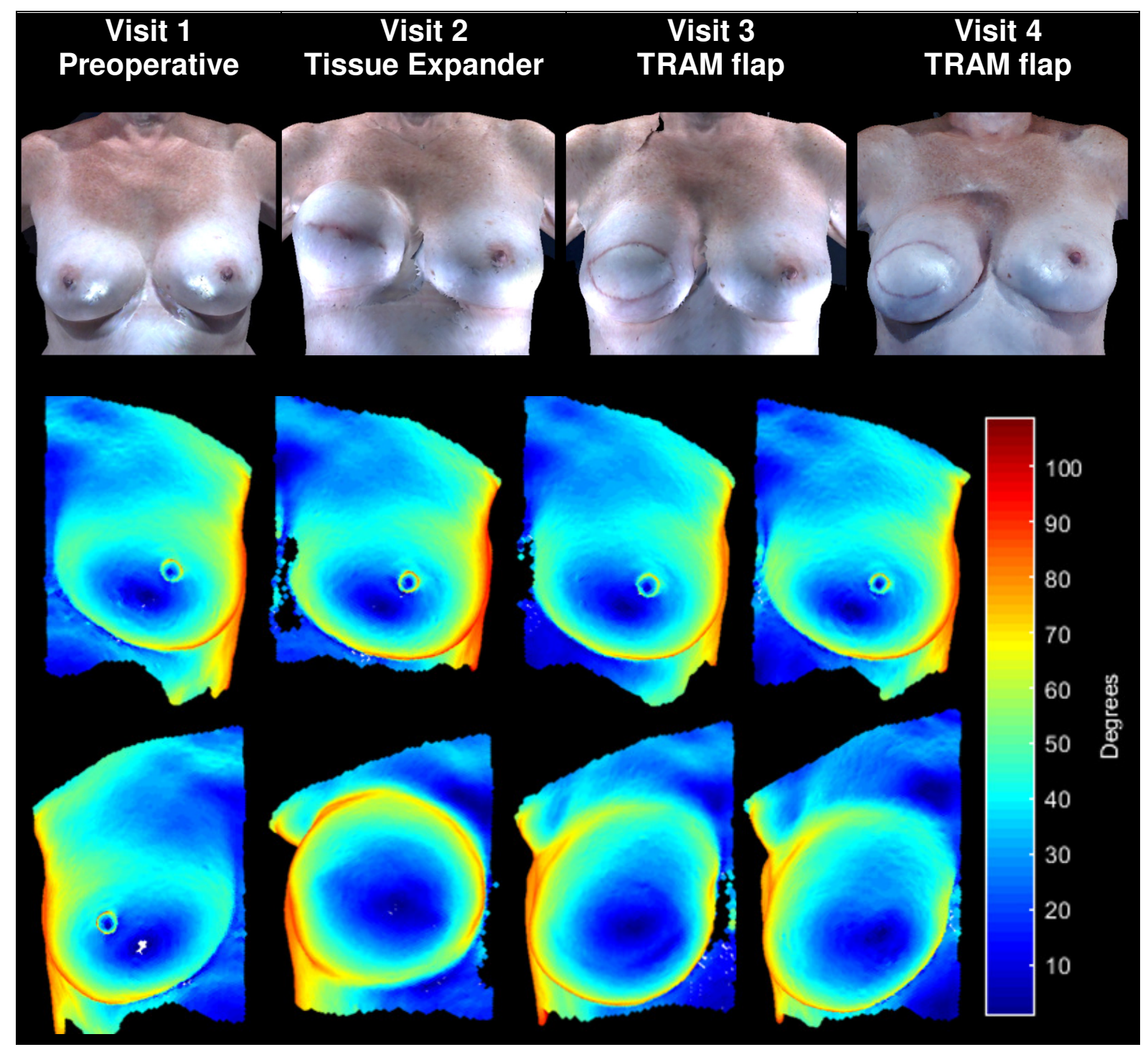

Fig. 4. Example of a patient who underwent TRAM flap reconstruction for the right breast while the left breast remained unoperated. The first row presents the $3 D$ photos of the patient at each visit. The second and third rows show the surface normal analysis of the left and right breasts, respectively. 


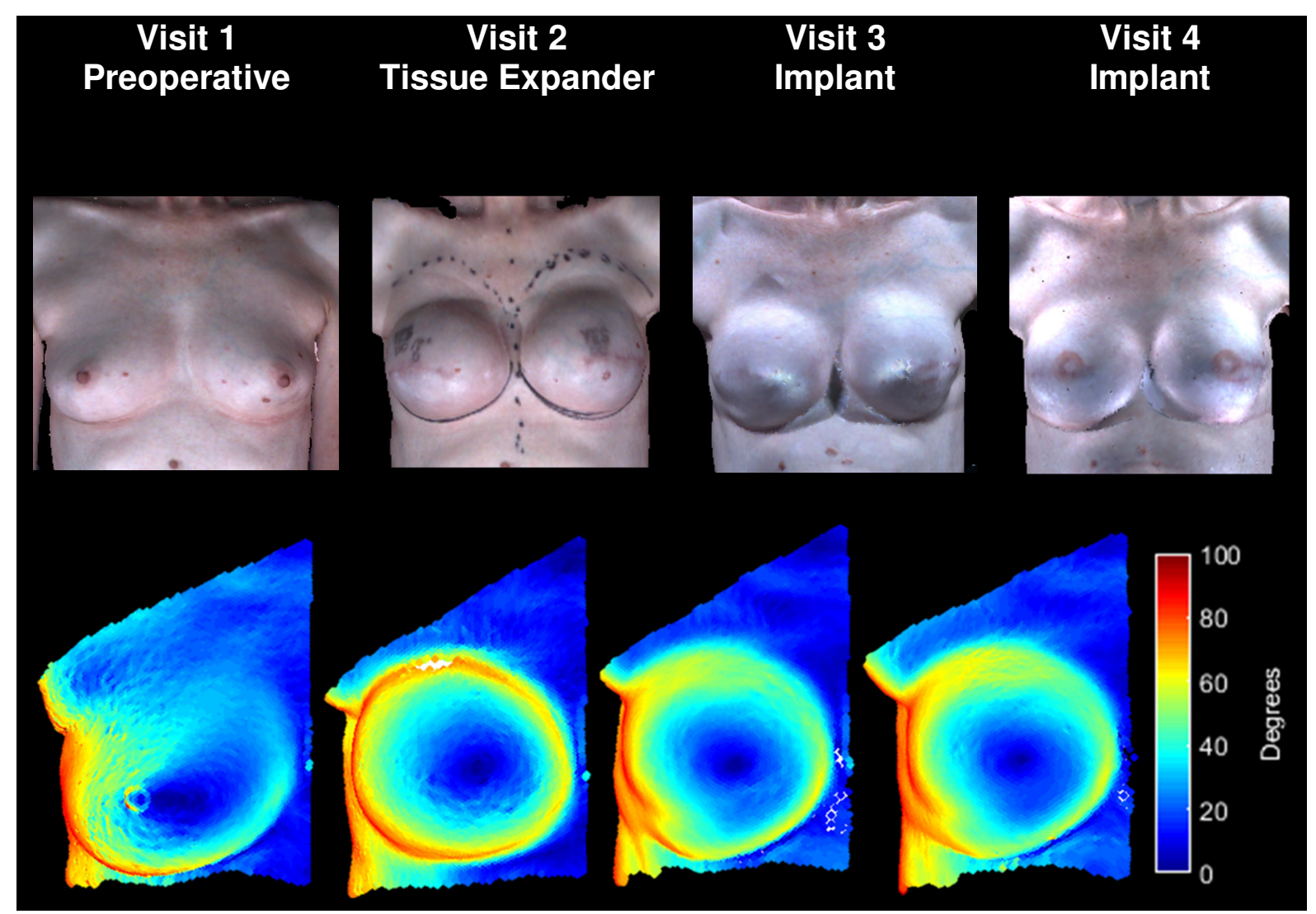

Fig. 5. Example of a patient who underwent bilateral implant-based reconstruction. The first row contains $3 D$ photos of the patient at each visit. The second row shows the surface normal analysis of the right breast.

Figure 6 presents bar graphs of the Bhattacharyya metric computed when comparing the overall shape of the breasts across the different visits for the five groups mentioned in Error! Reference source not found.. Figure $6 a$ (containing groups 1 (preoperative), 2 (TE to TRAM), and 3 (TE to implant)) presents the results of comparing the overall shape of the breast in visits 2-4 with the shape of the pre-operative breast at visit 1 (baseline). Figure $6 \mathrm{~b}$ is the same as Figure $6 \mathrm{a}$ except it presents the results for groups 1 (preoperative), 4 (TRAM w/o TE), and 5 (implant w/o TE). Both figures in Figure 6 show that the breast shapes of reconstructive groups are all significantly different $(p<0.05)$ from the preoperative group at every visit. Conversely, there are no significant differences in breast shape at all the stages between TRAM flap reconstruction and implant-based reconstruction.

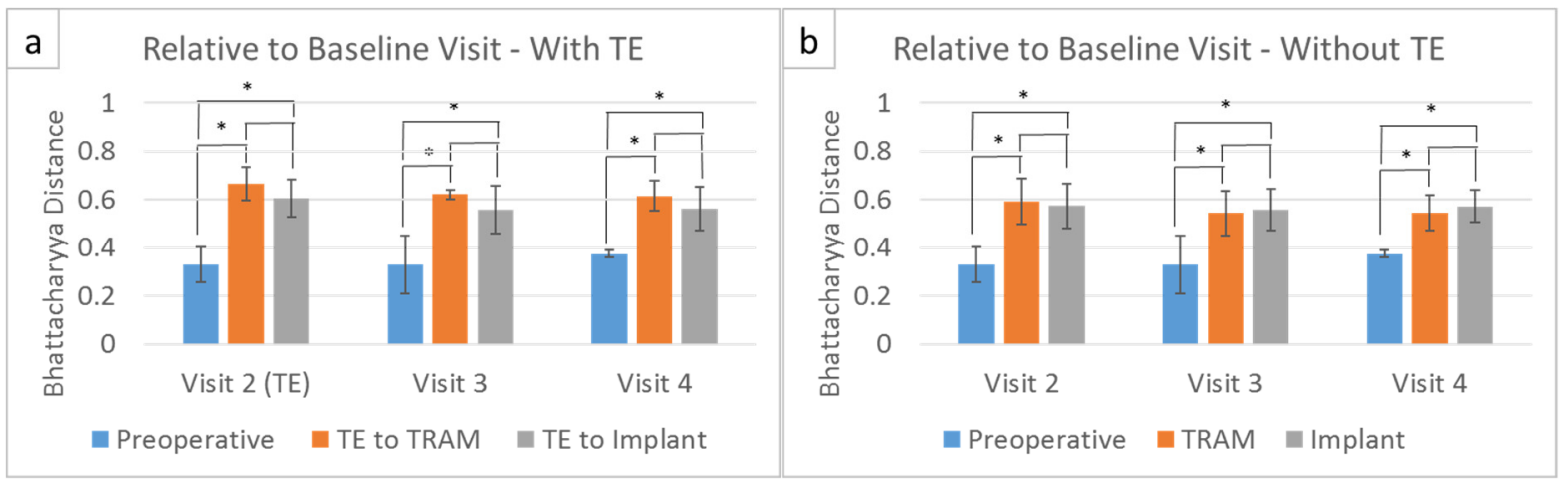

Fig 6. Bar graphs of the average and standard deviation Bhattacharyya distance of each visit to its baseline (first) visit. a) The first graph shows the comparison of the pre-operative breast images to the TE/TRAM and TE/Implant groups. The second visit represents the tissue expander stage and the third and fourth visits represent breast after the TRAM flap or implant exchange b) The second graph represents the TRAM flap or implant for visits 2 to 4 (tissue expander was not used). The black asterisks $\left(^{*}\right)$ indicate that the $t$-test $p$ values were less than 0.05 between the different groups. 
Results from the local shape analysis comparing the average surface orientation for each of the 32 subdivided regions across the different longitudinal stages of reconstruction (preoperative breasts, tissue expander placed within the breasts, TRAM flap reconstructed breasts, and implant-based breasts) are shown in Figure 7. The figure shows the sub-divisions of the breast and the four quadrants are color coded to represent UOQ (green), UIQ (blue), LOQ (red) and LIQ (orange). A black asterisk is shown in regions that indicate significant differences in local shape (t-test with $\mathrm{p}<0.05)$. For this analysis, patients were categorized into the TRAM flap group and implant group based on the timing of completion of the procedure such that each group contained images that were captured in the period of $3 \sim 6$ months following the surgical procedure. Most of the statistically significant differences occur in the upper-inner and lower-outer quadrants. As seen in the figure, the TE stage when compared to the Pre-Op group has the highest number of local regions (17) that show significant differences in shape. Next, the PreOp group compared to the implant group shows 10 local regions with significant differences in shape, followed by the TRAM flap reconstructed breasts showing about 7 local regions with significant shape differences when compared to the Pre-Op group. These results suggest that autologous reconstructions are relatively more similar in shape to preoperative breasts than breasts with implants. Interestingly, none of the regions show significant differences in local shape when comparing breasts with TE inserts to the breasts with implants, but there are 12 regions with significant differences in local shape when TRAM flap reconstructed breasts are compared to breasts with TE inserts. This data suggest that the local shape of breasts with implants is similar to the shape of breasts at the TE stage. Finally, several regions (10) show significant differences in local shape when comparing breasts with autologous reconstructions with those that have implants, suggesting that there are differences in localized shape of breasts in TRAM flap versus implants. Overall, these result conform with published studies [21, 22] indicating that autologous reconstructions are more amenable towards achieving a natural appearance.

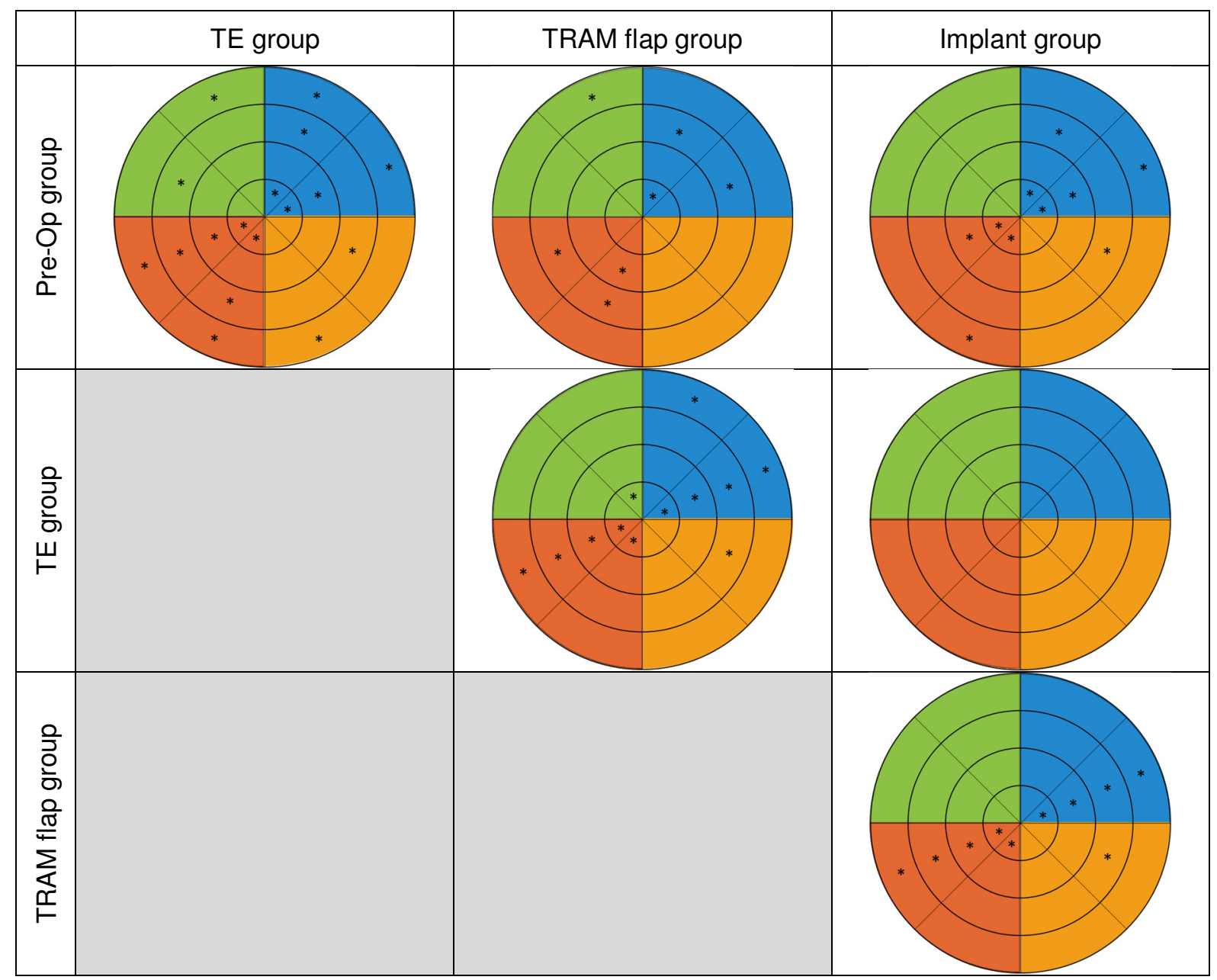

Fig. 7. Graphical representation of statistically significant regions between preoperative breasts (Pre-OP group), breasts with tissue expanders (TE group), TRAM flap reconstructed breasts (TRAM flap group), and implantbased reconstructed breasts (implant group). Sections marked with asterisks $\left(^{*}\right)$ are statistically significantly different between the two groups. The sections are color-coded based on Figure 3. 


\section{Discussion and Conclusion}

We demonstrated the use of surface normal orientation angles as a measure for analyzing longitudinally the morphological changes occurring in breast shape across the different stages of breast reconstruction surgery. Approaches were presented quantifying the overall shape of the breast and for estimating and visualizing localized changes in breast shape. Our approach is able to successfully quantify overall changes in breast shape across the different stages of reconstructive surgery from the preoperative shape to the shape after the completion of reconstruction. As expected, significant differences in the overall breast shape were measured when moving from the preoperative breast as it is surgically modified over the various stages of reconstruction. Maximal differences in breast shape occur when transitioning starting from the preoperative stage to the tissue expander stage and ending with the implant stage. Interestingly, the shape of the implant-based breasts is similar to the shape observed when tissue expanders are inserted, but there are differences in the shape of autologous reconstructed breasts when compared to breasts with implants. Also, although significant differences were not observed when comparing the shape of the final reconstructed breasts for both autologous and implant-based reconstructions to the preoperative breast shape, localized breast shape for autologous reconstructions has greater similarity with the preoperative breast shape than that of implant-based reconstructions.

It should be noted that the data were not adjusted to account for any changes in breast shape arising from certain non-surgical factors such as standing postures, arm positions, breathing, the menstrual cycle stage, image quality, and the manual extraction of the breast surfaces.

Further development of this method will help us compare shape differences across different individuals and evaluate breast shape symmetry. When evaluating breast symmetry, this method can help identify the local regions that may require revisions.

\section{Acknowledgements}

This material is based upon work supported by the National Science Foundation Graduate Research Fellowship under Grant No. DGE-1144207, and the National Institutes of Health under grant R01CA203984.

\section{References}

[1] J. T. Manning, D. Scutt, G. H. Whitehouse, S. J. Leinster, and J. M. Walton, "Asymmetry and the menstrual cycle in women," Ethol. Sociobiol., vol. 17, no. 2, pp. 129-143, 1996.

[2] D. Li, A. Cheong, G. P. Reece, M. A. Crosby, M. C. Fingeret, and F. A. Merchant, "Computation of breast ptosis from 3D surface scans of the female torso," Comput. Biol. Med., vol. 78, pp. 18-28, Nov. 2016.

[3] J. Lee, E. Kim, G. P. Reece, M. A. Crosby, E. K. Beahm, and M. K. Markey, "Automated calculation of ptosis on lateral clinical photographs," J. Eval. Clin. Pract., vol. 21, no. 5, pp. 900-910, 2015.

[4] M. J. Cardoso, H. Oliveira, and J. Cardoso, "Assessing cosmetic results after breast conserving surgery.," J. Surg. Oncol., vol. 110, no. 1, pp. 37-44, Jul. 2014.

[5] R. Zheng, W. Yu, and J. Fan, "Development of a new chinese bra sizing system based on breast anthropometric measurements," Int. J. Ind. Ergon., vol. 37, no. 8, pp. 697-705, 2007.

[6] C. Liu, J. Luan, L. Mu, and K. Ji, "The role of three-dimensional scanning technique in evaluation of breast asymmetry in breast augmentation: a 100-case study.," Plast. Reconstr. Surg., vol. 126, no. 6, pp. 2125-2132, 2010.

[7] D. K. Avşar, A. C. Aygit, E. Benlier, H. Top, and O. Taşkinalp, "Anthropometric breast measurement: a study of 385 Turkish female students.," Aesthet. Surg. J., vol. 30, no. 1, pp. 44-50, 2010.

[8] P. Agbenorku, M. Agbenorku, A. Iddi, E. Amevor, R. Sefenu, and D. Osei, "Measurements of breasts of young West African females: a guideline in anatomical landmarks for adolescent breast surgery.," Aesthetic Plast. Surg., vol. 35, no. 1, pp. 49-54, 2011.

[9] J. Lee, M. Kawale, F. a. Merchant, J. Weston, M. C. Fingeret, D. Ladewig, G. P. Reece, M. a. Crosby, E. K. Beahm, and M. K. Markey, "Validation of stereophotogrammetry of the human torso," Breast cancer basic Clin. Res., vol. 5, no. 1, pp. 15-25, Jan. 2011. 
[10]G. P. Reece, F. Merchant, J. Andon, H. Khatam, K. Ravi-Chandar, J. Weston, M. C. Fingeret, C. Lane, K. Duncan, and M. K. Markey, "3D surface imaging of the human female torso in upright to supine positions," Med. Eng. Phys., vol. 37, no. 4, pp. 375-383, Apr. 2015.

[11]H. R. Moyer, G. W. Carlson, T. M. Styblo, and A. Losken, "Three-Dimensional Digital Evaluation of Breast Symmetry after Breast Conservation Therapy," J. Am. Coll. Surg., vol. 207, no. 2, pp. 227232, 2008.

[12] A. Losken, I. Fishman, D. D. Denson, H. R. Moyer, and G. W. Carlson, "An Objective Evaluation of Breast Symmetry and Shape Differences Using 3-Dimensional Images," Ann. Plast. Surg., vol. 55, no. 6, pp. 571-575, 2005.

[13]M. Eder, F. V. Waldenfels, A. Swobodnik, M. Klöppel, A.-K. Pape, T. Schuster, S. Raith, E. Kitzler, N. a. Papadopulos, H.-G. Machens, and L. Kovacs, "Objective breast symmetry evaluation using 3-D surface imaging," The Breast, vol. 21, no. 2, pp. 152-158, 2012.

[14]M. Eder, M. Klöppel, D. Müller, N. a Papadopulos, H.-G. Machens, and L. Kovacs, "3-D analysis of breast morphology changes after inverted T-scar and vertical-scar reduction mammaplasty over 12 months.," J. Plast. Reconstr. Aesthet. Surg., vol. 66, no. 6, pp. 776-86, Jun. 2013.

[15]K. Ji, J. Luan, C. Liu, D. Mu, L. Mu, M. Xin, J. Sun, S. Yin, and L. Chen, "A prospective study of breast dynamic morphological changes after dual-plane augmentation mammaplasty with $3 \mathrm{D}$ scanning technique," PLoS One, vol. 9, no. 3, pp. 1-8, 2014.

[16] G. Catanuto, A. Spano, A. Pennati, E. Riggio, G. M. Farinella, G. Impoco, S. Spoto, G. Gallo, and M. B. Nava, "Experimental methodology for digital breast shape analysis and objective surgical outcome evaluation," J. Plast. Reconstr. Aesthetic Surg., no. 61, pp. 314-318, 2006.

[17] E. Van Limbergen and K. Van Tongelen, "Cosmetic evaluation of breast conserving treatment $f$ or mammary cancer. 1. Proposal of a quantitative scoring system," vol. 16, pp. 159-167, 1989.

[18]M. Kawale, J. Lee, S. Y. Leung, M. C. Fingeret, G. P. Reece, M. A. Crosby, E. K. Beahm, M. K. Markey, and F. A. Merchant, "3D symmetry measure invariant to subject pose during image acquisition," Breast Cancer Basic Clin. Res., vol. 5, no. 1, pp. 131-142, Jan. 2011.

[19]M. Eder, F. V. Waldenfels, M. Sichtermann, T. Schuster, N. a. Papadopulos, H. G. MacHens, E. Biemer, and L. Kovacs, "Three-dimensional evaluation of breast contour and volume changes following subpectoral augmentation mammaplasty over 6 months," J. Plast. Reconstr. Aesthetic Surg., vol. 64, no. June 2008, pp. 1152-1160, 2011.

[20] N. A. Thacker, F. J. Aherne, and P. I. Rockett, "The Bhattacharyya metric as an absolute similarity measure for frequency coded data," Kybernetika, vol. 34, no. 4, pp. 363-368, 1997.

[21]H. R. Teymouri, S. Stergioula, M. Eder, L. Kovacs, E. Biemer, and N. A. Papadopulos, "Breast reconstruction with autologous tissue following mastectomy," Hippokratia, vol. 10, no. 4, pp. 153162, 2006.

[22]S. J. Kronowitz and H. M. Kuerer, "Advances and surgical decision-making for breast reconstruction," Cancer, vol. 107, no. 5, pp. 893-907, 2006. 